\title{
Surfactant-free synthesis of mesoporous and hollow silica nanoparticles with an inorganic template $\dagger$
}

\author{
Luca Baù, ${ }^{* a}$ Barbora Bártová, ${ }^{b}$ Maria Arduini ${ }^{a}$ and Fabrizio Mancin ${ }^{a} a$ \\ Received (in Cambridge, UK) 27th August 2009, Accepted 16th October 2009 \\ First published as an Advance Article on the web 4th November 2009 \\ DOI: $10.1039 / b 917561 j$
}

A surfactant-free synthesis of mesoporous and hollow silica nanoparticles is reported in which boron acts as the templating agent. Using such a simple and mild procedure as a treatment with water, the boron-rich phase is selectively removed, affording mesoporous pure silica nanoparticles with wormhole-like pores or, depending on the synthetic conditions, silica nanoshells.

Mesoporous silica has been attracting increasing attention since the synthetic procedure based on surfactant templating was devised in 1992 by Beck and co-workers. ${ }^{1}$ By virtue of its high surface area and ease of functionalization, ${ }^{2}$ mesoporous silica is a unique material that holds promise for a host of applications, ranging from catalysis ${ }^{3}$ to sensing ${ }^{4}$ and, more recently, drug delivery. ${ }^{5}$ In recent years, considerable efforts have been devoted to the synthesis of mesoporous silica nanoparticles, combining the peculiar properties of silica nanoparticles, such as water solubility, biocompatibility, optical transparency and shorter diffusion paths, with the high loading capacity of mesoporous materials. ${ }^{6}$

The general approach involves, as in the case of mesoporous silica, the use of a templating agent, usually a surfactant, which is removed by calcination or chemical extraction after the silica network is formed. ${ }^{7}$ However, high temperature treatments may affect the properties of the silica particles, reducing the amount of surface silanol groups, ${ }^{8}$ and, in general, surfactant removal procedures are often incompatible with the presence of delicate payloads (drugs or other active species) inside the particles. Avoiding the use of surfactants is hence highly desirable and would be beneficial in terms of cost, environmental impact and scale-up potential. Furthermore, most synthetic procedures based on surfactant templating lead to ordered structures with non-interconnected cylindrical pores. Though undoubtedly crucial to many applications, these features are not always needed or even desirable. Other applications, like adsorption or catalysis, would benefit from the more accessible internal volume afforded by interconnected pores.

Surfactant-free synthesis of mesoporous silica can be performed using organic templates, ${ }^{9}$ but the removal procedures are similar to those used for surfactants. A different approach may involve the use of inorganic templates, as demonstrated by Asher and co-workers, ${ }^{10}$ who were able to tailor the morphology of growing silica nanoparticles using cadmium sulfide nanoparticles as templates. However, also in this case

\footnotetext{
${ }^{a}$ Dip. di Scienze Chimiche, Università di Padova, via Marzolo 1, 35131 Padova, Italy.E-mail: luca.bau@unipd.it, fabrizio.mancin@unipd.it

${ }^{b}$ CIME \& LSME, EPFL, Station 12, 1015 Lausanne, Switzerland

$\dagger$ This paper is dedicated to Professor Umberto Tonellato of

University of Padova on the occasion of his retirement.
}

harsh removal conditions, namely concentrated nitric acid treatment, were required.

We report here a novel surfactant-free approach to the synthesis of mesoporous nanoparticles in which the templating agent is a hydrolytically unstable inorganic phase, and the template removal treatment is a simple solvent exchange with water at room temperature. Such procedure results in the preparation of silica nanoparticles with interconnected disordered pores or, depending on the reaction conditions, silica nanoshells.

It has been known for several decades that silica glasses with nanometre-sized pores can be obtained by inducing phase separation in borosilicate glasses and selectively leaching the boron oxide phase. ${ }^{11}$ We reasoned that if a similar phase separation could be achieved in silica nanoparticles, a novel route to mesoporous nanoparticles could be envisaged.

In principle, such a separation should indeed occur. Silica nanoparticles may be easily prepared, according to the Stöber method, ${ }^{12}$ by condensation of alkoxysilane derivatives in ethanol-water-ammonia solutions at room temperature. The aqueous sol-gel synthesis of borosilicates, a similar procedure, has been extensively studied by Irwin and co-workers, ${ }^{13}$ who found the formation of borosiloxane $(\mathrm{Si}-\mathrm{O}-\mathrm{B})$ bonds to take place only upon dehydration at high temperatures. The absence of borosiloxane bonds points to the existence of boron-rich domains that should be easily removed by hydrolysis, leaving a network of pores in their place.

We set out to test this hypothesis by synthesizing borosilicate nanoparticles through a modified Stöber reaction, ${ }^{14}$ involving the co-condensation of silicon and boron oxide precursors. Tetraethoxysilane (TEOS) was used as a silicon source, while boric acid was chosen as the boron precursor in order to maximize the difference in the hydrolysis rates of the two precursors, which is known to correlate with oxide network inhomogeneity in sol-gel processes. ${ }^{15}$ The reaction was

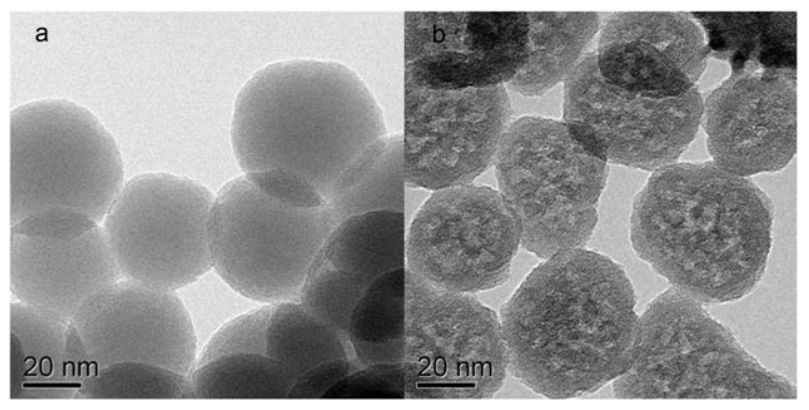

Fig. 1 TEM bright field images of borosilicate nanoparticles (a) and the same sample after water treatment (b). 
Table 1 Effect of water and ammonia on size ${ }^{a}$

\begin{tabular}{llll}
\hline Entry & {$\left[\mathrm{NH}_{3}\right] / \mathrm{M}$} & $\mathrm{H}_{2} \mathrm{O}(\%)$ & Size $/ \mathrm{nm}$ \\
\hline 1 & 0.30 & 6.8 & 50 \\
2 & 0.30 & 4.5 & 30 \\
3 & 0.30 & 2.3 & $-{ }^{a}$ \\
4 & 0.37 & 4.5 & 20
\end{tabular}

${ }^{a}$ No particles were isolated under these conditions after filtration over a $0.22 \mu \mathrm{m}$ PVDF filter.

Table 2 Effect of the $\mathrm{B} / \mathrm{Si}$ molar ratio in the reaction mixture on the boron content of the nanoparticles

\begin{tabular}{llll}
\hline Entry & $(\mathrm{B} / \mathrm{Si})_{\text {react }}$ & $(\mathrm{B} / \mathrm{Si})_{\text {prod }}$ & $\mathrm{B} \%(\mathrm{w} / \mathrm{w})$ \\
\hline 1 & 0.5 & 0.15 & 2.1 \\
2 & 0.75 & 0.12 & 1.7 \\
3 & 2 & 0.10 & 1.4 \\
\hline
\end{tabular}

monitored by dynamic light scattering $\ddagger$ until particle growth was complete. Unreacted species and hydrolyzed monomers were then removed by ultrafiltration with ethanol.

Size and morphology of the resulting borosilicate nanoparticles were investigated by TEM $\ddagger$. Spherical monodisperse particles, with a homogeneous interior and a smooth surface, are visible in the TEM images (Fig. 1a).

Monodisperse nanoparticles as small as $20 \mathrm{~nm}$ can be easily obtained by this method (see Table 1). Larger nanoparticles are obtained when a higher water concentration is used (entries 1 and 2). However, no nanoparticles can be isolated when the reaction mixture contains less than $4.5 \%$ water (entry 3). In order to obtain smaller particles, the ammonia concentration must be increased (entry 4).

The boron content was determined by spectrophotometric analysis using the azomethine $\mathrm{H}$ method ${ }^{16}$ and confirmed with ICP-OES analysisł (see Table 2). A 0.5 molar ratio of the boron and silicon precursors in the reaction mixture affords borosilicate nanoparticles with a boron content of $2 \%$ by weight. As observed also in the case of borosilicate glasses prepared by sol-gel synthesis, ${ }^{13}$ the use of higher $\mathrm{B} / \mathrm{Si}$ ratios in the preparation of the particles does not result in an increased boron loading.

The actual inclusion of boron into the nanoparticles was demonstrated by solution ${ }^{11} \mathrm{~B}$-NMR experimentsł̣. No ${ }^{11} \mathrm{~B}$ signal was detected when a nanoparticles sample was analyzed, because of the massive line broadening due to inclusion of the boron nuclei into the slowly diffusing nanoparticles. However, when the nanoparticles were dissolved by addition of concentrated $\mathrm{NaOH}$, boron derivatives were liberated in the solution and a sharp signal at $1.62 \mathrm{ppm}$, which can be assigned to the $\mathrm{B}(\mathrm{OH})_{4}^{-}$species, appeared.

The presence of boron is also confirmed by the $\mathrm{B}-\mathrm{O}$ absorption band at $1440 \mathrm{~cm}^{-1}$ in the FT-IR spectrum $\ddagger$ (Fig. 2). The lack of borosiloxane absorption bands (expected at 930 and $670 \mathrm{~cm}^{-1}$ ) suggests that, as expected, boron is not homogeneously distributed in the silica network but segregates into boron-rich domains. ${ }^{17}$

Encouraged by this finding, we tried to selectively leach the boron-rich phase. Nanoparticle solutions in ethanol were

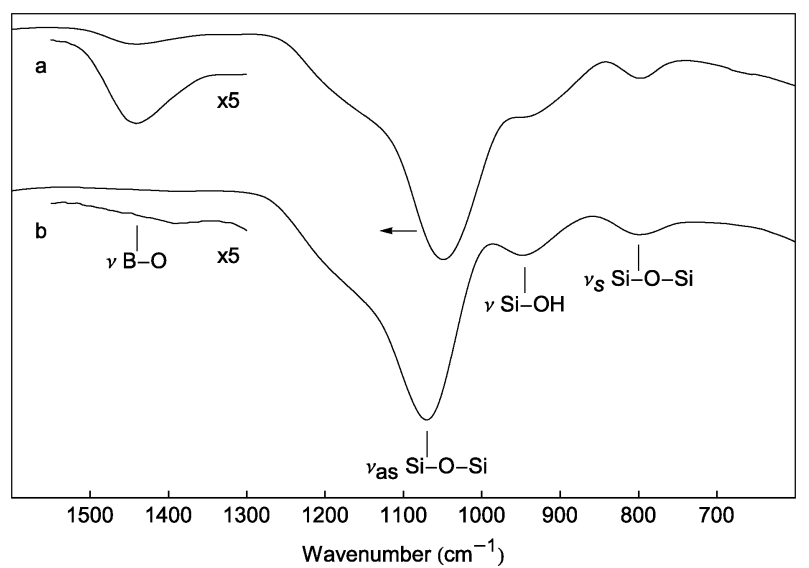

Fig. 2 FT-IR spectra images of borosilicate nanoparticles (a) and the same sample after water treatment (b).

extensively ultrafiltrated with water. After this treatment the B-O absorption disappears from the FT-IR spectrum and the complete leaching of boron is confirmed by ICP-OES analysis. The $\mathrm{Si}-\mathrm{O}-\mathrm{Si}$ stretching is blue-shifted from $1050 \mathrm{~cm}^{-1}$ to $1070 \mathrm{~cm}^{-1}$ (Fig. 2). Such shifts to higher frequencies are known to be associated with a decreased strain in the silica network $^{18}$ that would be consistent, in this case, with the removal of a templating phase. Remarkably enough, TEM micrographs of the nanoparticles after water treatment (Fig. 1b) show the formation of a pore network with a wormhole-like morphology, reflecting the distribution of the boron-rich phase in the parent borosilicate particles. The average size of the pores can be roughly estimated to lie in the $2-5 \mathrm{~nm}$ range. The rugged contour suggests slight surface erosion, but the particle size is not significantly decreased.

In a control experiment, pure silica nanoparticles were synthesized in identical conditions but in the absence of boric acid and subjected to the same water treatment. Samples taken before and after ultrafiltration look identical in TEM, and quite similar to the non-porous borosilicate particles as they appear before water treatment. This experiment confirms the role of boron as a templating agent, and excludes a possible effect of the water treatment alone on the formation of pores.

Interestingly, we also noticed that when the water content of the reaction mixture was increased to $6 \%$, a small fraction of hollow nanoparticles was present, along with the mesoporous particles, in the water-treated samples (Fig. 3). We decided to further investigate this phenomenon and found out that the fraction of nanoshells increases rapidly with the water content of the reaction mixture: when it reaches $10 \%$, most of the nanoparticles are hollow (Fig. 3).

This procedure paves the way to the realization of highly sophisticated silica nanosystems, where bulk doping, surface functionalization, pore wall functionalization and pore loading can be simultaneously exploited. The differential functionalization of pores and external surface of templated mesoporous silica is usually achieved by sequential treatment with different silane coupling agents before and after template removal. ${ }^{19}$ However, this procedure can result in promiscuous functionalization because of surfactant displacement. A diffusion-based approach that takes place after surfactant 


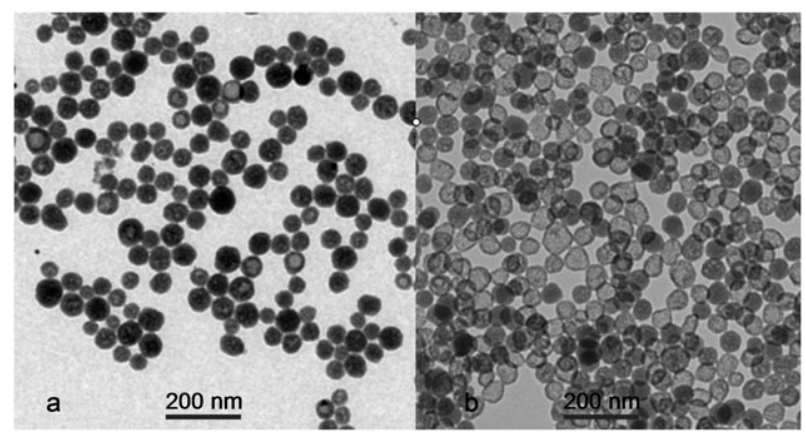

Fig. 3 TEM images of samples synthesized with increasing water content in the reaction mixture: (a) $6.8 \%$, (b) $10 \%$.

removal has been reported ${ }^{20}$ but is inherently limited to large nanoparticles where a precise control of the diffusion time is possible. Small borosilicate nanoparticles may be largely immune to template-silane exchange, and thus more suitable for selective functionalization.

In summary, we present a mild, surfactant-free method for the synthesis of hollow or mesoporous colloidal silica using boric acid as a templating agent. In contrast with the currently used methods for the preparation of similar system, the use of boric acid allows for an easy, cheap and mild removal of the template through a simple exposure to water. Moreover, tuning the water content in the reaction mixture allows the outcome of the reaction to be directed towards either mesoporous particles or nanoshells. Further investigations are under way to better characterize the system and explore the possible applications.

The authors would like to express their appreciation to Dr A. Sturaro and Dr R. Rella (Istituto per la Dinamica dei Processi Ambientali, CNR, Corso Stati Uniti 4, 35127 Padova) for skillful support in ICP-OES measurements, and to Mr Claudio Gamboz and Prof. Maria Rosa Soranzo (University of Trieste) for some TEM measurements.

\section{Notes and references}

$\ddagger$ Transmission electron microscopy (TEM) images were acquired using a Philips/FEI CM300 machine operated at $300 \mathrm{kV}$ (CIME, EPFL) and a Philips EM208 machine operated at $100 \mathrm{keV}$ (CSPA, University of Trieste). Samples were sonicated and a few drops were deposited on holey carbon copper grids or standard carbon-coated copper grids (200 mesh). Dynamic light scattering (DLS) measurements were obtained with a Malvern Zetasizer Nano S instrument. Infrared spectra were obtained in attenuated total reflectance mode on a Nicolet 5700 FT-IR instrument. ${ }^{11}$ B NMR spectra were obtained on a Bruker Avance DRX 300 spectrometer. ICP-OES analyses were performed with a Varian Liberty Series II instrument. Samples for ICP-OES were mineralized in $6 \mathrm{M} \mathrm{NaOH}$ at $70^{\circ} \mathrm{C}$ for one hour prior to analysis.
Typical synthesis of borosilicate nanoparticles. To a thermostated vessel charged with a solution of boric acid $(13 \mathrm{mg}, 0.21 \mathrm{mmol})$ in ethanol $(20 \mathrm{~mL}), 100 \mu \mathrm{L}(0.45 \mathrm{mmol})$ of TEOS were added under stirring at $25{ }^{\circ} \mathrm{C}$. $0.6 \mathrm{~mL}$ of milliQ water and $0.4 \mathrm{~mL}$ of a $14.8 \mathrm{M}$ aqueous solution of ammonia were then added to initiate the polymerization. After 16 hours, the solution was filtered through a $0.22 \mu \mathrm{m}$ PVDF filter. The solution was then diluted to $80 \mathrm{~mL}$ with ethanol and concentrated to the original volume by ultrafiltration through a regenerate cellulose membrane (cut-off $10 \mathrm{kDa}$ ) under nitrogen pressure $(4 \mathrm{bar})$. The procedure was repeated five times. The resulting solution was filtered through a $0.22 \mu \mathrm{m}$ PVDF filter.

Synthesis of mesoporous silica nanoparticles. The borosilicate nanoparticles solution was diluted to $80 \mathrm{~mL}$ with water and concentrated to the original volume by ultrafiltration through a regenerate cellulose membrane (cut-off $10 \mathrm{kDa})$ under nitrogen pressure $(4 \mathrm{bar})$. The procedure was repeated five times. The resulting aqueous nanoparticles solution was then filtered through a $0.22 \mu \mathrm{m}$ PVDF filter.

1 C. T. Kresge, M. E. Leonowicz, W. J. Roth, J. C. Vartuli and J. S. Beck, Nature, 1992, 359, 710.

2 F. Hoffmann, M. Cornelius, J. Morell and M. Fröba, Angew. Chem., Int. Ed., 2006, 45, 3216.

3 C. Crudden, M. Sateesh and R. Lewis, J. Am. Chem. Soc., 2005, 127, 10045; D. J. Mihalcik and W. Lin, Angew. Chem., Int. Ed., 2008, 47, 6229 .

4 A. Descalzo, D. Jimenez, M. Marcos, R. Martínez-Máñez, J. Soto, J. E. Haskouri, C. Guillém, D. Beltrán, P. Amorós and M. Borrachero, Adv. Mater., 2002, 14, 966.

5 N. K. Mal, M. Fujiwara and Y. Tanaka, Nature, 2003, 421, 350; S. Giri, B. G. Trewyn, M. P. Stellmaker and V. S.-Y. Lin, Angew. Chem., Int. Ed., 2005, 44, 5038.

6 Selected reviews: (a) I. I. Slowing, B. G. Trewyn, S. Giri and V. S. Y. Lin, Adv. Funct. Mater., 2007, 17, 1225; (b) C. Barbe, J. Bartlett, L. G. Kong, K. Finnie, H. Q. Lin, M. Larkin, S. Calleja, A. Bush and G. Calleja, Adv. Mater., 2004, 16, 1959.

7 J. Kobler, K. Möller and T. Bein, ACS Nano, 2008, 2, 791.

8 C. Yague, M. Moros, V. Grazu, M. Arruebo and J. Santamaria, Chem. Eng. J., 2008, 137, 45.

9 Y. Wei, D. Jin, T. Ding, W. Shih, X. Liu, S. Z. D. Cheng and Q. Fu, Adv. Mater., 1998, 10, 313.

10 S.-Y. Chang, L. Liu and S. A. Asher, J. Am. Chem. Soc., 1994, 116, 6739 .

11 H. P. Hood and M. E. Nordberg, US Pat., $2106744,1934$.

12 W. Stöber, A. Fink and E. Bohn, J. Colloid Interface Sci., 1968, 26, 62.

13 A. Irwin, J. Holmgren, T. Zerda and J. Jonas, J. Non-Cryst. Solids, 1987, 89, 191.

14 An alternative procedure for the synthesis of larger borosilicate nanoparticles was recently reported in V. K. Parashar, J.-B. Orhan, A. Sayah, M. Cantoni and M. A. M. Gijs, Nat. Nanotechnol., 2008, 3, 589 .

15 C. J. Brinker and G. Scherrer, Sol-Gel Science, The Physics and Chemistry of Sol-Gel Processing, Academic, Boston, 1990.

16 D. L. Harp, Anal. Chim. Acta, 1997, 346, 373.

17 The degree of condensation of boron-rich domains (boron oxide, borates or other condensed forms) is unclear at this level of investigation.

18 (a) R. A. B. Devine, J. Non-Cryst. Solids, 1993, 152, 50; (b) A. Agarwal and M. Tomozawa, J. Non-Cryst. Solids, 1997, 209, 166 .

19 F. de Juan and E. Ruiz-Hitzky, Adv. Mater., 2000, 12, 430.

20 K. Cheng and C. C. Landry, J. Am. Chem. Soc., 2007, 129, 9674. 